\title{
A Review of Blood Flow Through Micro Channel
}

\author{
Nitesh Pudke ${ }^{1}$, Nalini Nagpure ${ }^{2}$, Bhola Rode $^{3}$, Sahil Yadao $^{4}$, Tripti Kumbalpuri ${ }^{5}$ \\ ${ }^{1,2,3,4,5}$ Department of Mechanical Engineering, Government College of Engineering, Nagpur 441108, India
}

Received on: 11 December, 2020, Revised on: 11 January, 2021 , Published on: 16 January, 2021

\begin{abstract}
The microfluidic platform has increasingly been explored for research, engineering and biological application. Microfluidic is the major study of microcirculation in which microfluidic refers to the behavior, precise control and manipulation of fluids that are geometrically constrained to a small scale. Microfluidic devices are expected to provide tool not only to better understand the biophysical behavior of blood flow in micro vessels but also for disease diagnosis. In this review, we provide an overview of blood flow through the micro channel. A special emphasis on obtaining the steady flow characteristics of blood using micro-PIV (particle image velocimetry) and the most practical and the recently developed methods for microfluidic device fabrication. Numerical and computational methods needed to analyze fluid characteristics are also introduced.
\end{abstract}

Keywords - Micro fluids, Micro channel, Micro-PIV, Fabrication methods.

\section{I-INTRODUCTION}

$\mathbf{U}$ nderstanding the fluid mechanics inside the human body has been one of the high motivators of the systematic examine of fluid flow, in general, since the very early days. However, in recent years, due to advances in computers, optics, and digital image processing techniques, it has become possible to combine a conventional particle image velocimetry (PIV) system with an inverted microscope and consequently improve both spatial and temporal resolution. The present review outlines the most relevant studies on the flow properties of blood at a micro scale level by using past video-based methods and current micro-PIV and confocal micro-PIV techniques. More recently, with the advent of sophisticated experimental and analysing techniques and due to the ease and controllability over experimental parameters, scientists turned to in-vitro models to address bio fluid mechanics inside the vessels. Although the comparability of results between in-vitro studies and the invivo or ex-vivo studies remains debatable, they still prove to be extremely useful for understanding the basic flow physics as well as for the design and development of novel biomedical devices. The major challenges associated with in-vitro investigations are miniaturization, complex nature of the fluid itself, and the deformability of the vessel wall. With the advent of fabrication technologies like photolithography and soft lithography, miniaturization is now possible even in the range of nanometres. The complexity of fluids like blood can be addressed by using blood analog fluids that can mimic its mechanical properties Additionally the most recent computational fluid dynamics studies on micro scale hemodynamic are also 


\section{International Journal of Innovations in Engineering and Science, www.ijies.net}

reviewed by R. Lima [1] who acquire pace measurements for several optically sectioned images along the depth of the micro channel the use of confocal micro particle photograph velocimetry (PIV). Also Raj M [2] used similar micro-PIV (particle image velocimetry) to pick out the problematic components of the pressure as well as the rate area. Gale [3] indicates a unique way to evaluate where he emphasis on both the most realistic and these days advanced methods for microfluidic tool fabrication. He specially copes with laminate, moulding, 3-d printing, and high resolution nanofabrication techniques. The techniques are compared for his or her relative costs and advantages, with unique interest paid to the commercialization prospects of the diverse technology. Microfluidics affords a brilliant possibility to create gadgets able to outperforming classical strategies in biomedical and chemical studies Neil Convey and Nikolaj Gadegaard [4] proposed a paper where they confirmed the progress and review on 30 years of microfluidics where they appraise how factors inclusive of government investment encouraged the development of latest substances and fabrication strategies. Cutting-edge packages of microfluidics are also examined and they highlight regions where paintings need to be focused within the destiny to ensure that the era realizes its complete potential. Microfluidic platforms have an increasing being explored for in vitro blood diagnostics and for reading complex micro vascular tactics where D. Pasias [5] in modern- day year obtained pace fields the usage of microPIV techniques. The analytical answer for blood speed within the channel is advanced utilizing the power law version for blood viscosity. The consequences confirmed that RBC aggregation has an impact on the past due stages of the flow, located particularly within the bluntness of the speed profiles. On the initial levels of the waft, the shearing conditions are determined moderately improved, preventing excessive RBC mixture formation. Because the drift decelerates in the channel, RBC aggregation will increase, affecting the drift characteristics.

This study motivates to cope with the aforementioned troubles in the existing experimental paradigm of small deformation studies in deformable micro channel with a non- Newtonian fluid flow, author investigated FSI in physiologically applicable embedded deformable micro channel with varying softness the use of polydimethylsiloxane (PDMS). Such PDMS-primarily based phantoms have won plenty attention recently due to the benefit of fabrication and operation, optical compatibility, biocompatibility, and because of the reality that the rheological parameters of this elastomer can be tuned very without problems via converting the base to cross-linker ratio for the duration of preparation. Furthermore, disease applicable functions like stenosis also can be included easily in such systems. Cylindrical micro channel with a size compare to arteries were fabricated the use of a frugal procedure utilizing a pull-out smooth lithography approach. The popular in-vitro blood mimicking fluid Xanthan gum (XG) solution is used as the non-Newtonian bio fluid. Unique attention is dedicated to the cautious characterization of both the deformable solid wall and the non-Newtonian fluid. Stress drop and wall deformation are monitored as a characteristic of the imposed drift rate. Moreover, velocity fields had been evaluated to look at the impact of the deformable wall on the velocity distribution. A simple mathematical version is also proposed to assess the flow triggered deformation of the channel wall.

The relaxation of the paper is prepared as follows: Sec. II \& sec. III explains the material used and method fabrication of the micro channel, sec. Iv flow visualization techniques, In Sec. V, a simple mathematical formulation is developed to. The salient conclusions of this study are summarized in Sec. VI.

\section{II-DIFFERENT FABRICATION MATERIAL FOR MICROCHANNEL}

\section{A) Polymeric and glass substrates}

In the last few years, microfluidic devices have been started to be manufactured on polymeric substrates instead of silicon and glass substrates mainly because of their low cost. The low cost also allows them to be manufactured as a disposable device. The foremost important polymeric materials for microfluidic devices are polymethylmethacrylate (PMMA) and polydimethylsiloxane (PDMS). in recent years, PMMA has come up as a solution to wide range of microfluidic devices because of its low cost and associated easier fabricating methods. PMMA is perfectly transparent and is used as a substitute for glass. It is often used as a drug delivery system material, implants and possesses high biocompatibility. It also produces lesser amount of heat- 


\section{International Journal of Innovations in Engineering and Science, www.ijies.net}

affected zone (HAZ) when compared to other polymer materials such as polycarbonate (PC) and polypropylene (PP) when processed under laser processing methods. The microfluidic channels on PMMA can also be manufactured precisely using hot embossing. PMMA has been found to be most easily fabricated substrate. Materials using advanced femtosecond laser processing.

Glass was used as an alternative to silicon for many microfluidic applications. Glass was superseded as the standard microfluidic material by cheaper alternatives that would allow for a simple approach to the manufacture of microfluidic while still allowing for the interpolation of valves and pump. Towards the late $20^{\text {th }}$ century the elastomeric material polydimethylsiloxane (PDMS) pioneered by George Whiteside and his group at Harvard, it quickly became the most popular material for the manufacture of microfluidic devices. Compared to glass and silicon, the fabrication of devices in PDMS is simple and does not require expensive clean room facilities. Firstly, a master structure is prepared (through silicon micromachining) next, the PDMS base and curing agent are mixed together before the solution is poured over a mould. The low surface energy of the PDMS means that it readily flows into small features and release from the mould is simple. Another main advantage of PDMS is how it can be bonded to itself on to other materials. PDMS channel can be sealed through a variety of methods. In the simplest method, tape can be used to seal channels but it is more common place for devices to be sealed against a glass slide or with a further layer of PDMS. When placed in conformal contact with another substrate, the elastomeric nature of PDMS means that is forms a seal capable of withstanding moderate fluid pressure. PDMS polymer have some useful properties, including good optical transparency and biocompatibility, easily reversible sealing to glass, elasticity, replication of fine and complex geometries, permeability to glass, thermal stability and low cost. Because of these properties this material was suitable for studying several phenomena in the microcirculation by combining it with our confocal micro PIV system.

Some other polymeric materials have also been studied such as PC,28 polystyrene (PS)29 and polyethylene terephthalate (PET).30 Some authors have also studied cyclic olefin polymers such as Topas31 or Zeonor.32 Polymers offer high chemical resistance and good optical properties besides offering a range of possibilities for functionalization and are cheap to process.

Fabricating micro channel on glass poses a big challenge for conventional fabricating techniques. The process requires a series of steps. This makes the whole process cost to become significant. However, fabricating micro channel on glass substrates is a time- consuming process. However, because of high degree of transparency, inertness to most substances and ability to sustain higher temperature make it first choice for most of the microfluidic device [4].

\section{B) Semiconductors, ceramics and composites}

Most of the early developments in micro channel-based applications were based on silicon and were originally developed for integrated circuit (IC) industry. The highspeed digital circuits are required to be cooled at faster rate for performing better with millions of logic gates built over it. For cooling of such devices, either forced air convection technology or liquid-based micro- channel technology is used. However, liquid cooling has been proved to be far much efficient than forced air convection cooling. Silicon is not only used for micro- electronic devices but also applications such as fuel cells. Apart from silicon, quartz is also widely used as a substrate material for several micro channel-based devices. Quartz is sometimes more suitable than silicon for these purposes because it is (1) chemically inert and stable, (2) optically transparent, (3) cheaper than silicon and (4) a good electrical insulator [8] Gallium nitride $(\mathrm{GaN})$ is another important semiconductor material which has attracted considerable interest over recent years because of wide band gap and can be used in various optical devices including light emitting diode(LED) and laser diode. Photo structured glass ceramic (Foturan) has also been used in three-dimensional (3D) microfluidic devices. Photo structured glass ceramic was developed by Schott Glaswerke and is optically transparent to human eyes. It has been specially developed for 3D microfluidic devices. In recent years, ceramic-based heat exchangers have also been investigated. Although the high manufacturing cost associated with ceramic heat exchangers often pose a challenge, some new fabrication technologies have been developed in recent times for manufacturing of ceramic-based heat exchangers. Pressure laminated integrated structure (PLIS) method developed by Kee et al. offers a cost-effective solution for such ceramicbased devices 


\section{International Journal of Innovations in Engineering and Science, www.ijies.net}

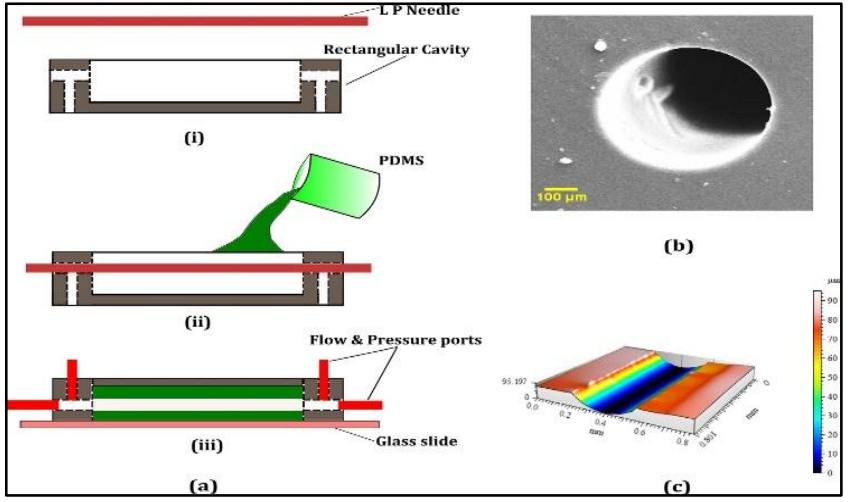

Fig. 1- Steps involved in the fabrication of the cylindrical micro channel and their characterization. (a) (i) The needle mold and rectangular brass cavity. (ii) Pouring PDMS into the cavity with the mold in position. (iii) Mold removed after the PDMS in cured and a base glass slide and ports inserted. (b) SEM of the crosssection of the channel. (c) $3 D$ Profilometry of the inner channel surface along a longitudinal cut section. (adapted from [2])

\section{III-METHODS OF FABRICATING MICROCHANNEL}

The ability to coax a wide variety of materials into even smaller device is based on progress in micromachining and other fabrication techniques. To date, different types of fabrication methods for different types of materials and applications have been studied by different researchers across the world and the list is very exhaustive. Although there are some methods such as lithography, 3D printing and laser micromachining which have been discussed widely, there are some methods that are very uncommon and very few literatures are available. In the following sections, some of the most used fabrication processes have been discussed briefly.

\section{A) Soft lithography}

Above fig.1. shows Soft lithography. It been developed as an alternative to Photolithography and a replication technology for both micro and nanofabrication. In soft lithography, LP (Lumbar puncture) needles of nominal diameter, $\mathrm{D}=510$ micrometer (Comet India), were used as the replicating mold for the fabrication process. A rectangular brass cavity of dimensions (30 - $15-5 \mathrm{~mm})$ was fabricated to hold the PDMS micro channel and to house the inlets, outlets, and the pressure sensing ports. The micro channel fabricated inside the cavity is of length $\mathrm{L}$ which is taken as the distance between the inlet and outlet pressure sensing terminals. The base polymer and curing agents of the two-part PDMS were mixed in the required ratio and kept in a vacuum chamber to remove air bubbles generated during mixing. For the current experiment, two base to cross linker ratios were chosen, 10:1 (CH10) and 30:1(CH30). The PDMS mixture was then poured over the needle mold which was kept along the length from inlet to outlet as shown in Figure and cured in an oven at $95 \mathrm{C}$ for 12 -h. The needle is gently pulled off from the PDMS matrix once it is cured and the inlet, outlet, and pressure sensor ports were inserted into the structure using hollow metallic needles. The current method is frugal compared to many other soft lithography techniques as it does not employ any mold fabrication process like photolithography or any other rapid prototyping methods. Furthermore, the present fabrication technique yielded very good results regarding surface homogeneity and withstood high pressures during leak flow testing. The final dimension of the cross-section of the micro channel was observed under a microscope and the diameter was measured using standard image processing methods.

\section{B) $3 D$ printing}

3D printing was utilizing to produce a micro-mold for a PDMS microfluidic device. ABS plastic was selected because it is one of most commonly used thermoplastics for FDM technique, and it is reactive to surface modification with the use of an AVB (acetone Vapour Bath). The design is first modelled with the desired parameters using the $3 \mathrm{D}$ CAD software and then the file is saved. The file is then saved again, as a. STL file, this file format is supported by many software packages which are used for rapid prototyping and 3D printing. In order to print the mold, the previously saved. STL file is first exported into the corresponding software of the 3D printer. The 3D printing process for the micro-mold is illustrated in fig. 2. Figure shows the design of the fluidic layer which consists of three micro-channels and a reservoir. Two of the micro-channels act as the vessels for the fluid inlets which is directed towards the mixing reservoir that is situated in the centre of the device. A third micro-channel, which acts as the vessel for the fluid outlet, is directed from the mixing reservoir towards the fluid outlet. The fluidic layer mold has a 


\section{International Journal of Innovations in Engineering and Science, www.ijies.net}

channels height of $0.75 \mathrm{~mm}$ and width of $2 \mathrm{~mm}$. It also has a check valve assist with a height of $0.25 \mathrm{~mm}$.

\section{C) Laser-Evolved Micro channeling}

As far as process complexity is concerned, laser associated micro channel fabrication processes are most simple in nature with capability of generating almost every shape of microstructures. Laser processing is very versatile, takes minimum amount of time and can be used on almost every type of substrate material. In most of the cases, the material removal phenomenon is heat dependent; the processing must be optimized for getting the best possible result.

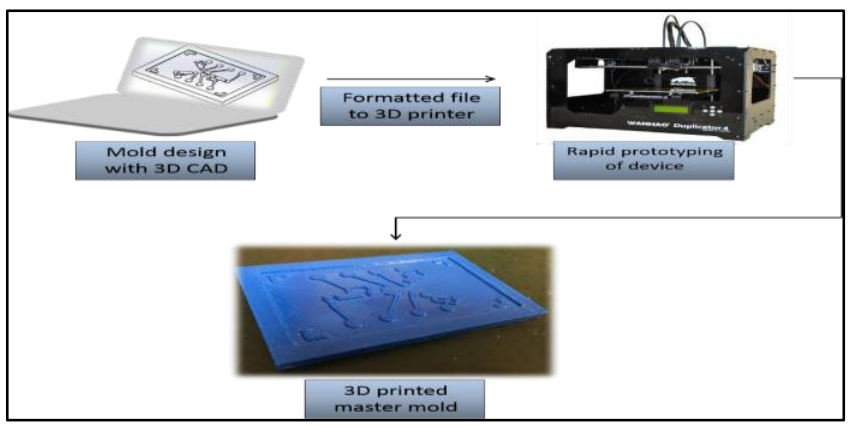

Fig. 2 The 3D printing process for the micro-mold (adapted from [18])

Laser processing of micro channel has evolved recently and has been studied by different researchers. In most of the researches, either neodymium doped yttrium aluminum garnet (Nd: YAG) or excimer laser has been used. A few researchers have also used $\mathrm{CO} 2$ lasers for fabricating micro channel. Most of the laser fabricating processes involve either one or maximum two steps only; they do not require clean room facilities. They do not require the preparation of mask, such as in photolithographic process or etching process. There are numerous advantages of using lasers as micro channeling tool over other processes. Laser-based processes are environmentally clean, scrap less and based on simple-to-operate techniques. With the use of appropriate kind of laser, almost every kind of material including metals, non-metals and ceramic can easily be cut into almost any shape. It also covers a wide range of operations such as drilling, engraving, scribing, joining, micro structuring and surface modification which can be used to fabricate the whole device in its entirety. Recently, micro fabrication using laser-assisted photo polymerization technique has enabled the Micro features as small as 120 $\mathrm{nm}$ on urethane acrylate monomer. IN the market, there are different kinds of laser sources available producing wavelengths ranging from deep UV (157 nm) to IR (10mm) and pulse durations from microseconds to femtoseconds.

\section{IV - FLOW VISUALISATION TECHNIQUES IN MICROCIRCULATION}

Measuring blood flow in a major blood vessels are nonoptical methods measurement are Doppler ultrasound and magnetic resonance imaging. The non-optical method of measurement of flow suffers from the disadvantage of being invasive. Whereas later comes under optical method and are of non-invasive nature [6] Doppler ultrasound and MRI techniques too have several drawbacks. One of them is inability to obtain quantitate detailed information at a micro scale level due to the relatively poor spatial resolution [7].

In the past laser Doppler imaging were used for flood flow measurement in micro vessels. Results obtained are in the form of direct phometric information without image information.

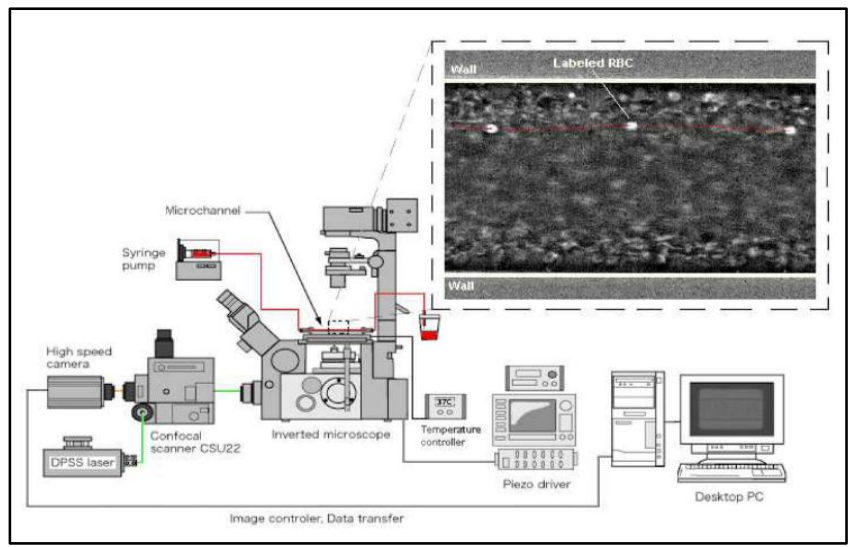

Fig. 3 Confocal micro-PTV system used to measure the flow of labelled RBCs at both diluted and high suspensions of blood cells (adapted from [7])

However, with the advancements in measurement techniques video-based methods are widely used and hence given reliable results in the mentioned literatures [1,2] $\mathrm{A}$ very recent video-approach to study the flow properties of blood in microcirculation is the micro-particle velocimetry. Due to its high spatial and temporal resolution.

Lima and his colleagues [1] Investigated the ability of a confocal micrp-piv system to measure the velocity profiles 


\section{International Journal of Innovations in Engineering and Science, www.ijies.net}

of both physiological saline (PS) and in vitro blood (20\% Hct) in rectangular (300 $\mu \mathrm{m}$ wide, $45 \mu \mathrm{m}$ deep) polydimethylsiloxane (PDMS) micro channel. Generally, the velocity profiles were found to be markedly blunt in the central region, mainly due to the low aspect ratio $(\mathrm{h} / \mathrm{w}=0.15)$ of the rectangular micro channel. Predictions using a theoretical model for the rectangular micro channel corresponded quite well with the experimental micro-PIV results for the PS fluid. However, for the in vitro blood with $20 \%$ Hct, small fluctuations were found in the ensemble velocity profile most likely due to the interactions between neighboring RBCs, high shear rate generated in the vicinity of the walls and temporal fluctuations of the local Hct. similarly, Raj met al [2] Visualized flow by the micro-PIV in which author used two micro channel of varying base to cross linker ratio. The synchronizer of the micro-PIV is synced with the pressure sensor with the TTL signal from the Lab view interface for the simultaneous measurement of pressure drop and the velocity field. An iterative multi-pass cross-correlation algorithm using Fast Fourier Transform (FFT) with a final sub-window size of $32 \times 32$ pixel and an overlap of $50 \%$ was chosen to evaluate the final velocity filed. Additionally, this work also demonstrated that confocal micro-PIV can be effectively integrated with a PDMS micro channel and used to obtain blood velocity profiles along the full depth of the micro channel because of its unique 3-d optical sectioning ability.

Particle-Based Flow Diagnostic Techniques in Micro-PIV To tract the fluid movement different tracer particle of varying size $(\sim \mu \mathrm{m}$ in size) used in various studies.

With the assumptions: -

- Particle tracer moves with the same velocity as local flow velocity

- Tracer particle should be neutrally buoyant and small enough to follow the flow perfectly.

- Tracer particles should be big enough to scatter the illumination lights efficiently.

- It should not alter the flow properties.

Also, refractive index of the particle to that of the final should be taken in consideration.

Flow through Micro channel

The perfusion of blood in micro channel devices is typically achieved through pressure driven set-up for studying flow characteristics. The flow should be steady and uniform which is generally done by syringe pump [2] An alternate flow driven option i.e. surface tension driven blood flow to examine the aggregating and non-aggregating $\mathrm{RBC}$ and newt anion suspension in a rectangular glass micro channel was examined by [5]. Surface tension driven (STD)blood flow offers a simpler and cost effective option to the pressure driven flows. Surface tension is a material property of a fluid-fluid interface whose origins lie in the different attractive intermolecular force that acts in the two

fluid phases. STD flow depends on the hydrophobicity/hydrophobicity of the surface material. Hydrophilic materials have good affinity with fluids resulting in droplet formation.

\section{V- SMATHEMATICAL MODELLING OF BLOOD FLOW THROUGH RECTANGULAR MICROCHANNEL BY TWO FLUID APPROACHES}

The blood is assumed to behave as a two-component mixture comprised of plasma and red blood cells (RBCs). The plasma is assumed to behave as a viscous fluid whereas the RBCs are given a granular-like structure where the viscosity also depends on the shear-rate [9].

For the two phase formulation two methods are widely used Mixture Theory (or the theory of interacting continua) [11] and Averaging Method(s) [10]. Both approaches are based on the underlying assumption that each component may be mathematically described as a continuum. Here we use mixture theory for numerical modelling [13].

Let plasma component is represented by $\mathrm{S} 1$ and the $\mathrm{RBC}$ component by S2, X1 and X2 denote the positions of $\mathrm{S} 1$ and $\mathrm{S} 2$ in the reference configuration. The motions of the constituents are represented by the mappings $\left(\chi 1\right.$ and $\left.\chi^{2}\right)$. The motion of the two components can be represented as [16] [9]

$$
\boldsymbol{x}_{1}=\chi_{1}\left(\boldsymbol{X}_{1}, t\right), \boldsymbol{x}_{2}=\chi_{2}\left(\boldsymbol{X}_{2}, t\right)
$$

while the kinematical quantities associated with the motions take the expressions

$$
\begin{gathered}
\boldsymbol{v}_{1}=\frac{d_{1} \boldsymbol{x}_{1}}{d t}, \quad \boldsymbol{v}_{2}=\frac{d_{2} \boldsymbol{x}_{2}}{d t} \\
D_{1}=\frac{1}{2}\left(\frac{\partial v_{1}}{\partial x_{1}}+\left(\frac{\partial v_{1}}{\partial x_{1}}\right)^{T}\right), \quad D_{2}=\frac{1}{2}\left(\frac{\partial v_{2}}{\partial x_{2}}+\left(\frac{\partial v_{2}}{\partial x_{2}}\right)^{T}\right)
\end{gathered}
$$




\section{International Journal of Innovations in Engineering and Science, www.ijies.net}

Where, $v$ is the velocity field, $D$ is the symmetric part of velocityy gradient, and $\mathrm{d} 1 \mathrm{dt}$ and $\mathrm{d} 2 \mathrm{dt}$ denote differentiation with respect to time holding $X_{1}$ and $X_{2}$ fixed, respectively.

Assuming no interconversion of mass between the two components, conservation of mass for the plasma and the RBCs take the form The bulk density field, $\rho_{1}$ and $\rho_{2}$, for these two components are

$$
\rho_{1}=(1-\phi) \rho_{10}, \rho_{s}=\phi \rho_{20}
$$

where $\rho_{10}$ and $\rho_{20}$ are the pure density of plasma, and the $\mathrm{RBCs}$, in the reference configuration; $\phi$ is the volume fraction of RBCs, the haematocrit, where $0 \leq \phi<\phi_{\max }<1$.

In the absence of thermo-chemical and electromagnetic effects, the governing equations consist of the conservation of mass, linear momentum and angular momentum. The

$$
\frac{\partial \rho_{1}}{\partial t}+\operatorname{div}\left(\rho_{1} \boldsymbol{v}_{1}\right)=0
$$

equations of conservation of mass in the Eulerian form are [15] [12],

$$
\frac{\partial \rho_{2}}{\partial t}+\operatorname{div}\left(\rho_{2} \boldsymbol{v}_{2}\right)=0^{(4.2)}
$$

where $\partial \partial \mathrm{t}$ is the derivative with respect to time and div is the divergence operator, while the equations of balance of the linear momentum are given by,

$$
\begin{gathered}
\rho_{1} \frac{D^{1} \boldsymbol{v}_{1}}{D t}=\operatorname{div}\left(\boldsymbol{T}_{1}\right)+\rho_{1} \boldsymbol{b}_{1}+\boldsymbol{f}_{l}(5.1) \\
\rho_{2} \frac{D^{2} \boldsymbol{v}_{2}}{D t}=\operatorname{div}\left(\boldsymbol{T}_{2}\right)+\rho_{2} \boldsymbol{b}_{2}-\boldsymbol{f}_{l}(5.2)
\end{gathered}
$$

where in general for any scalar $\beta, \mathrm{D} \alpha \beta \mathrm{Dt}=\partial \beta \partial \mathrm{t}+\mathrm{v} \alpha \cdot \nabla \beta, \alpha=$ $1, \quad 2$, and (for any vector $w$ ), D $\alpha \mathrm{wDt}=\partial \mathrm{w} \partial \mathrm{t}+(\nabla \mathrm{w})$ $\mathrm{v} \alpha, T_{1}$ and $T_{2}$ stand for the Cauchy stress tensors, $f_{I}$ represents the interaction forces (exchange of momentum) between the components, and $b_{1}$ and $b_{2}$ refer to the body force. $T_{1}, T_{2}$ and $f_{I}$ will be given by the constitutive equations.

Constitutive Equations

\section{A) Stress tensors for plasma}

We assume that the plasma behaves as a linear viscous fluid [12]

$$
\boldsymbol{T}_{1}=\left[-p_{1}+\lambda_{1} \operatorname{tr} \boldsymbol{D}_{1}\right] \boldsymbol{I}+2 \mu_{1} \boldsymbol{D}_{1}
$$

where $p_{1}$ is the plasma pressure, $\mu_{1}$ and $\lambda_{1}$ are the first and second coefficients of viscosity of the plasma, 'tr' is the trace operator, and $I$ is the identity tensor.

\section{B) Stress tensor for RBCs}

We model the RBCs as a generalized Reiner-Rivlin model proposed by [12], which exhibits a shear dependent viscosity and includes the effect of porosity,

$$
\boldsymbol{T}_{2}=\left[\beta_{1}+\beta_{2} \operatorname{tr} D_{2}\right] \boldsymbol{I}+\beta_{3} D_{2}+\beta_{4} D_{2}^{2}
$$

where $\beta_{1}, \beta_{2}, \beta_{3}$ and $\beta_{4}$, depend on the haematocrit $\phi$;

Interaction Forces:

For the interaction forces, we use a simplified form of the constitutive equation proposed by Johnson, Massoudi, and Rajagopal (1990) and Massoudi (2003),

$$
f_{I}=A_{1} \nabla \phi+A_{2} F(\phi)\left(v_{2}-v_{1}\right)+A_{3} \phi\left(2 \operatorname{tr} D_{1}^{2}\right)^{-1 / 4} D_{1}\left(v_{2}-v_{1}\right)
$$

where the first term represents the force due to the density gradient (Muller, 1968); the second term is related to the (Stokes) drag force; and the third corresponds to the shear lift (or Saffman's lift [17] force. Furthermore, $A_{1}, A_{2}$ and $A_{3}$ are related to material properties, $F(\phi)$ is called the hindrance function which comes from the generalization of the interaction force from a single particle (RBC) to an assembly of particles. It can be obtained from the empirical correlations of sedimentation of particles [12]

$$
A_{2}=\frac{9 \mu_{1}}{2 a^{2}}, \quad A_{3}=\frac{3(6.46)\left(\rho_{1} \mu_{1}\right)^{2}}{4 \pi a}
$$

Advance in Microscale Numerical Studies:

Numerical methods appropriate for microscale blood flows; a boundary element method, immersed boundary methods, and particle methods [14] 


\section{International Journal of Innovations in Engineering and Science, www.ijies.net}

\section{A) Boundary element method}

The RBC can be modelled by a capsule with a thin membrane and containing Newtonian fluids. The flow around a RBC is assumed to be a Stokes flow. In using a capsule model, the flow field inside and outside a capsule can be described by the Stokes equation and the continuity equation, and the problem is closed with equilibrium and constitutive equations describing a mechanical behaviour of the membrane. One of the most successful numerical method for addressing this problem is a boundary element method (BEM) first developed by Youngren and Acrivos.

\section{B) Immersed Boundary Method}

An elegant method of fluid-structure coupling is the immersed boundary method (IBM). The IBM was originally developed by Peskin for simulating blood flow in the heart, and the method was extended to a front-tracking method by Univerdi and Tryggvason. In the IBM, fluids inside and outside capsule are treated as a single-phase fluid.

\section{C) Practical Method}

Mesh free particle methods are also suitable for microscale hemodynamic problems. In the particle methods, both the membrane of capsules and the internal and external fluids are represented by the cluster of particles. The particle method tracks the front of membrane using the membrane particles.

\section{VI-CONCLUSION}

In this review paper we have explained the different methods of blood flow through micro channel. Various conventional engineering method and advancement in fabrication of micro channel like 3-D printing is also demonstrated. Varieties of material where used for fabrication but most preferable by many mentioned literatures was PDMS (polydimethylsiloxane) because of unique characteristics which best suited for the diagnostics and research work in biological field. Confocal micro-PIV setup is most reliable for visualizing the steady flow characteristics of blood flow through micro channel. Numerical model and new advancement in the recent years is also explained in this paper.

\section{RESEARCH GAP}

Past result and conclusion of various research paper on blood flow behavior in micro scale, where it behaves as a non-Newtonian fluid, we have found several issues like fabrication and modeling of channels at micro scale, poor spatial resolution, optical errors arisen from walls of channels, high concentration of blood cells, difficulty in visualization of results due to insufficient computing power and reliable image analysis techniques. In our studies we are trying to sort out above problems by experimental and analytical analysis .

In experimental work we are going to fabricate micro channel by PDMS polymer which have many useful properties mentioned in material of our study and we will be finding flow visualization of blood which is flowing through micro channel with the help of latest measuring computer technique i.e. PIV (particle image velocimetry) with inverted microscope. Then we analyze the results come from experimental work with the analytical modeling by software MATLAB, ANSYS etc.

By analyzing the experimental and analytical part of our work we will make easy understanding of not only blood but also the other non-Newtonian fluid flow behavior in micro scale and application in microfluidic, biomedical, electrical appliances etc.

\section{REFERNECES}

[1] Rui Lima, Shigeo Wada, Shuji Tanaka, Motohiro Takeda, Takuji Ishikawa, Ken-Ichi Tsubota, Yohsuke Imai, Takami Yamaguchi. Biomed Microdevices (2008) 10:153-167

[2] Kiran Raj M, Jeevanjyoti Chakraborty, Sunando Dasgupta and Suman Chakraborty.Flow-Induced Deformation in A Microcahnnel with A Non-Newtanion Fluid. Biomicrofluidics 12, 034116 (2018)

[3] Bruce K. Gale, Aleaxandar R. Jafek, Christopher J. Lambert, Brady L. Goenner, Hossein Moghimifan, Ugochukwu C. Nze and Suraj Kumar Kamarapu. A Review of Current Methods in Microfluidic Device Fabrication and Future Commercialization Prospects. Inventions 2018,3,60 


\section{International Journal of Innovations in Engineering and Science, www.ijies.net}

[4] Neil Convery and Nikolaj Gadeguard.30 Years of Microfluidics Micro and Nano Engineering 2 (2019) 7691

[5] D. Pasias, A. Passos, G. Constantinides, S, Balabani and E. Kalivotis. Surface Tension Driven Flow Of Blood In A Rectangular Microfluidic Channel:Effect Of Erythrocyte Aggreagation. Phys. Fluids 32, 0719030

[6] A.K. Jayanthy, N. Sujatha and M. Ramasubba Reddy. Measuring Blood Flow: Techniques and Application - A Review. IJRRAS 6 (2), 2011

[7] R. Lima, T. Ishikawa, Y. Imai and T. Yamaguchi. Bllod Flow Behavior in Microchannels: Past, Current and Future Trends. January 2012

[8] Shashi Prakash and Ubrata Kumar. Fabrication of Microchannels: A Review. Enginnering Manufacture 2015.

[9] Mehrdad Massoudi, James f. Antaki. Computational study of Microchannel. June 2015.

[10] Ishii M. Thermo-fluid dynamic theory of two-phase flow. NASA STI/Recon Technical Report A. 1975; $75: 29657$.

[11] Rajagopal K, Tao L. Mechanics of Mixtures, Series on Advances in Mathematics for Applied Sciences, vol. 35. Singapore: World Scientific; 1995.

[12] Wei-Tao WU, Nadine Aubry, Mehrdad Massoudi, Jeongho Kim, and James F.Antaki A Numerical Study of Blood Flow Using Mixture Theroy.[ Int J Eng Sci. 2014 Mar 1; 76: 56-72].

[13] Wei-Tao WU, Fang Ang,Nadine Aubry, Mehrdad Massoudi, Jeongho Kim, and James F.Antaki Study of blood flow in several benchmark microchannel using a two fluid approach.[ Int J Eng Sci. 2015 Oct 1; 95: 49-59].

[14] Rui A. Lima Study of Flow Behaviour in Microchannel:Past, Current and Future Trend [ January 2015].

[15] Atkin RJ, Craine RE. Continuum theories of mixtures: Applications. IMA Journal of Applied Mathematics . 1976a;17(2):153-207.

[16] Johnson G, Massoudi M, Rajagopal KR. Flow of a fluidsolid mixture between flat plates. Chemical Engineering Science. 1991;46(7):1713-1723.
[17] Saffman P. The lift on a small sphere in a slow shear flow. Journal of Fluid Mechanics. 1965;22(02):385-400.

[18] Carlton McMullen. Design, Fabrication, and Testing of a 3D PrinterBasedMicrofluidic System"(2015). 